\title{
Silicon Nanocolumns on Nanosphere Lithography Templated Substrates: Effects of Sphere Size and Substrate Temperature
}

\author{
Christian Patzig ${ }^{1, *}$, Bodo Fuhrmann², Hartmut S. Leipner ${ }^{2}$, and Bernd Rauschenbach ${ }^{1}$ \\ ${ }^{1}$ Leibniz-Institut für Oberflächenmodifizierung e.V., Permoserstraße 15, 04318 Leipzig, Germany \\ ${ }^{2}$ Interdisziplinäres Zentrum für Materialwissenschaften, Martin-Luther-Universität Halle, \\ Heinrich-Damerow-Straße 4, 06120 Halle, Germany
}

\begin{abstract}
Glancing angle ion beam sputter deposition was used to grow regular arrays of Si nanocolumns with a nominal height of $650 \mathrm{~nm}$ at room temperature on polystyrene nanospheres with sphere diameters between $260 \mathrm{~nm}$ and $3550 \mathrm{~nm}$, and at elevated temperatures on $\mathrm{SiO}_{2}$ nanospheres with a sphere diameter of $360 \mathrm{~nm}$. Top view and cross sectional scanning electron microscopy reveals that the Si nanocolumns resemble cylinder-like structures, terminated by a hemispherical cap. Diameter, height and inter-column-spacing are found to depend linearly on the nanosphere diameter, thus giving the possibility to grow arrays of vertical $\mathrm{Si}$ columns with distinct porosities. For the growth at elevated temperatures, it was found that while on non-patterned substrates diffusion effects lead to broadening and finally merging of initially separated nanocolumns, on nanosphere patterned substrates this broadening effect is only moderate. No merging of columns is observable in this case, but a decrease of the column height due to a temperature-driven inter-column densification.
\end{abstract}

Keywords: Silicon, Nanocolumns, Nanoshores, Lithography.

\section{INTRODUCTION}

The oblique- or glancing angle deposition (GLAD) process $^{1}$ is a physical vapor deposition technique used to create highly underdense films that consist of arbitrarily shaped nano- and microstructures. Making use of a highly oblique incidence angle $\beta$ of the particle flux that impinges the substrate (usually $\beta>80^{\circ}$ measured to the substrate normal), self-shadowing at the atomic level occurs during the initial stages of film growth. As the first islands and clusters that grow on the substrate surface cast shadows in the direction opposite to the incoming particle beam, they prevent the following flux to reach those shadowed zones, which in turn fosters the growth of a film that consists of rod-like shaped nanostructures, slanted towards the direction of the incoming particle flux. With the use of a suitable substrate rotation, unique and almost arbitrarily shaped nanostructures, such as nanopillars, nanospirals, and zigzag structures can be grown., ${ }^{2,3}$ Potential applications of those nanosculpted films include humidity sensors, ${ }^{4}$ polarizing filters, ${ }^{5}$ photonic crystals, ${ }^{6}$

${ }^{*}$ Author to whom correspondence should be addressed. magnetic storage devices, ${ }^{7}$ pressure sensors ${ }^{8}$ and supports for enzyme immobilization. ${ }^{9}$

While the GLAD growth on unpatterned substrates results in randomly distributed nanostructures that tend to broaden with increasing height, ${ }^{10}$ a patterned substrate will provide specific nucleation sites for the particle flux, thus enabling the growth of uniform nanostructures with distinct periodicity over the substrate surface. Patterning techniques to provide arrays of nucleation sites for GLAD include electron beam lithography (EBL) $)^{3,11}$ and nanosphere lithography (NSL), in the latter either using the nanospheres as shadowing mask for a subsequent evaporation or etching process ${ }^{12}$ in order to establish an array of nucleation sites, or using the nanospheres themselves as hexagonally closed packed (hcp) arranged template. ${ }^{2}$

In this paper, the GLAD growth of vertical $\mathrm{Si}$ nanocolumns on polystyrene (PS) nanospheres, arranged in a two-dimensional hcp pattern, of different sphere diameter $D$ ( $D$ ranging from $260 \mathrm{~nm}$ to $3550 \mathrm{~nm})$ will be discussed. The diameter $w$, height $h$, inter-column-spacing $d$ and morphological structure of the Si nanocolumns will be examined as function of $D$. It will be shown that $w$, $h$ and $d$ scale linearly with $D$, thus enabling the possibility 
to grow $\mathrm{Si}$ nanocolumns with pre-defined aspect ratios and inter-column spacings. Additionally, the effect of an increased substrate temperature $T_{\mathrm{S}}$ on the growth of $\mathrm{Si}$ nanocolumns on $\mathrm{SiO}_{2}$ nanospheres with $D=360 \mathrm{~nm}$ will be shown and discussed.

\section{EXPERIMENTAL DETAILS}

$5^{\prime \prime} \mathrm{Si}(111)$ wafers (Si-Mat), $(625 \pm 25) \mu \mathrm{m}$ thick, As-doped with a resistivity of $(0,001-0,006) \Omega \mathrm{cm}$, were cut into $2 \times 2 \mathrm{~cm}^{2}$ pieces. Immediately prior to usage, the substrates were cleaned by immersion in a solution of $25 \%$ $\mathrm{NH}_{4} \mathrm{OH} / 30 \% \mathrm{H}_{2} \mathrm{O}_{2} / \mathrm{H}_{2} \mathrm{O}$ (1:1:5) for $10 \mathrm{~min}$ at $80{ }^{\circ} \mathrm{C}$ and thoroughly rinsed with Milli-Q water (Millipore Simplicity). PS particles (10 wt \% aqueous suspension) and $\mathrm{SiO}_{2}$ particles (5 wt $\%$ aqueous suspension) of different diameters were obtained from Microparticles, Berlin, Germany.

Self assembly of the PS particles was achieved by a method similar as described in Ref. [13]. A petri dish was filled with Milli-Q water and a cleaned glass slide was partially immersed into the water under an angle of $30^{\circ}$. Then the PS particle suspension (diluted 1:1 with ethanol) was transferred onto the water surface by dropping it onto the glass slide. When the particle suspension on the glass slide flowed down and reached the water surface, unordered monolayers formed. By adding a drop of a surfactant, e.g., 2\% sodiumdodecylsulfate, a hexagonal close packed arrangement of the spheres was achieved. Finally, the masks were transferred to the prepared substrates.

$\mathrm{SiO}_{2}$ particle suspensions were diluted 1:3 with Milli-Q water and spin coated onto the silicon substrates with spin speeds ranging from 400 to $600 \mathrm{rpm}$.

Figure 1(a) shows an example of a patterned substrate prior to deposition.

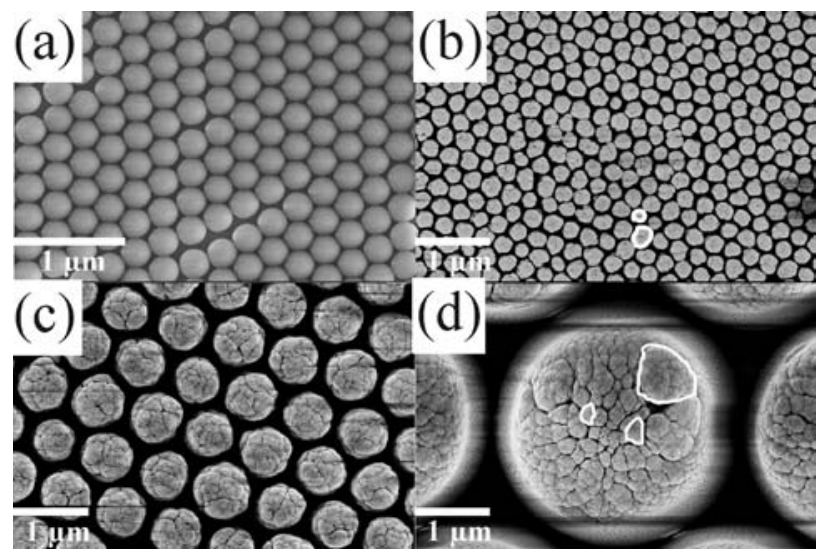

Fig. 1. (a) Example of NSL-patterned substrate with $D=260 \mathrm{~nm}$. (b)-(d) Top view micrographs of Si nanocolumns grown on (b) $D=$ $260 \mathrm{~nm}$, (c) $D=760 \mathrm{~nm}$ and (d) $D=3550 \mathrm{~nm}$. Some of the single $\mathrm{Si}$ nanorods that contribute to the whole nanocolumn on spheres with $D=$ $3550 \mathrm{~nm}$ are highlighted in (d). Columns with small and big diameters on spheres with $D=260 \mathrm{~nm}$ are highlighted in (b).
The $\mathrm{Si}$ nanocolumns were grown in a stainless steel load-locked vacuum system with a base pressure of approximately $3.0 \times 10^{-9}$ mbar. The experimental setup as described elsewhere ${ }^{3}$ consists of an inductively coupled, high-frequency $(13.56 \mathrm{MHz})$ ion source with a triple grid system of $40 \mathrm{~mm}$ in diameter. $\mathrm{Ar}^{+}$-ions with an energy of $1100 \mathrm{eV}$ were extracted out of the source to sputter the surface of a sintered polycrystalline Si disc $(99.99 \%$ pure, positioned $15 \mathrm{~cm}$ in front of the ion source) at an angle of $\phi_{\text {Target }}=65^{\circ}$ to the target normal. An $\mathrm{Ar}^{+}$-flux of $5.5 \mathrm{sccm}$ resulted in a working pressure of $9.5 \times 10^{-5}$ mbar. The substrates were attached to a molybdenum substrate holder that allows for a continous variation of the incoming angle of particle flux $\beta$, that is, the angle between target and substrate normal. Additionally, the substrate can be rotated around the substrate normal with different angular speeds $\omega$ and can be heated using a tantalum wire resistance heater at the back side of the substrate holder. The temperature was monitored and controlled with a type $\mathrm{K}$ thermocouple attached to the substrate holder's back side. The temperature at the substrate surface could be estimated from the thermocouple temperature as described in a previous work. ${ }^{14}$

The sputtered Si flux reached the substrate, positioned $12 \mathrm{~cm}$ in front of the target, under an angle $\beta=85^{\circ}$, yielding a nominal vertical growth rate $r \approx 4.3 \mathrm{~nm} / \mathrm{min}$ on unpatterned substrates. For all experiments, the angular speed was set to $\omega=0.2 \mathrm{rev} / \mathrm{min}$, in order to resemble a circular symmetric particle flux. The deposition time was set to $150 \mathrm{~min}$, according to a nominal Si column height $h_{0}$ of $650 \mathrm{~nm}$ on unpatterned substrates at room temperature (RT). All growth experiments on substrates patterned with PS nanospheres of diameter $D=260,500,760,2550$ and $3550 \mathrm{~nm}$ were performed at RT. The experiments on the substrates patterned with $\mathrm{SiO}_{2}$ nanospheres $(D=360 \mathrm{~nm})$ were performed at $T_{\mathrm{S}}=\mathrm{RT}, 150{ }^{\circ} \mathrm{C}$ and $350{ }^{\circ} \mathrm{C}$, with a deposition time of $162 \mathrm{~min}$, yielding $h_{0}=700 \mathrm{~nm}$ on unpatterned substrates at RT.

After growth, the samples were cleaved and studied using scanning electron microscopy (SEM) with $2.5 \mathrm{kV}$ acceleration voltage. To obtain micrographs that show a good compositional contrast of the Si nanocolums and the underlying nanospheres, back-scatter electron imaging was used in some cases.

The analysis of the micrographs in terms of diameterand inter-column-spacing determination was done using the commercially available Scanning Probe Image Processor ${ }^{15}$ version 3.2.6.0. (SPIP) with the grain detection modules. The mean values $\langle w\rangle$ of the Si nanocolumn diameters were evaluated as follows: First, SPIP with the grain detection module was used on top view micrographs showing a sufficiently large number of columns to analyze their diameter values. Then, a Gaussian distribution plot with those diameter values was done for every nanosphere diameter size $D$ (see inset of Fig. 3(b) as an example). 


\section{RESULTS AND DISCUSSION}

\subsection{GLAD on PS Nanospheres with Different Sphere Diameters $D$}

Figures 1(b-d) show top view micrographs of $\mathrm{Si}$ nanocolumns grown on hcp arranged nanospheres with different sphere diameters $D$. As can be seen, the hcp arrangement of the underlying self-assembled nanosphere layer is perfectly adopted by the $\mathrm{Si}$ columns grown on it. However, the morphology of the columns changes with increasing sphere diameter. For $D=260 \mathrm{~nm}$, single columns evolve out of one sphere that serves as nucleation site. Those columns have mean diameters $\langle w\rangle=235 \mathrm{~nm}$. The distribution of the $w$ values is rather broad, and columns with diameters ranging from $w \approx 130 \mathrm{~nm}$ to $w \approx$ $300 \mathrm{~nm}$ can be found. To underline this, two columns with small and big diameters are highlighted in Figure 2(b). For $D=3550 \mathrm{~nm}$, Figure 1(d) shows that several single nanorods, each one having diameters between $150 \mathrm{~nm}$ and $900 \mathrm{~nm}$, start to grow on top of the PS sphere. The "whole" column is made of approximately 50 of those nanorods and has a mean diameter of $\langle w\rangle=3310 \mathrm{~nm}$. However, the distribution of $w$ is less broad for those bigger PS sphere diameters. Table I lists the values for $\langle w\rangle$, the standard deviation $\sigma$ of the Gaussian distribution and the normalized standard deviation $\sigma /\langle w\rangle$. Obviously, there are two trends observable with increasing nanosphere diameter $D$. The first trend can be seen as a change of the growth mode from single-column growth on spheres having small $D$ values to multi-column growth on spheres having larger diameters $D$. This trend is attributed to the fact that on PS spheres with small $D$, only a few nuclei will form on each nanosphere. The probability for sufficient internuclei-competition will be small, resulting in a collective growth of nanorods that form on the nuclei and grow together to form a single nanocolumn on each sphere. ${ }^{2}$ On PS spheres with large $D$ values, more nuclei will form in the beginning of the growth process, increasing the probability for inter-nuclei competition on one sphere. Some of the nuclei will capture more flux than the others (for example, because of local inhomogenities in the deposition flux), thus quickly evolving into nanorods that shadow the surrounding nuclei and increase their diameter with increasing deposition time. Thus, as the growth continues, several nanocolumns will evolve on one PS sphere with sufficiently large $D$ and will finally contribute to the "big" nanocolumn that grows on the PS sphere.

The second trend is the increased homogeneity of the mean nanocolumn diameter $\langle w\rangle$ with increasing $D$. Table I shows that the normalized standard deviation gets almost bisected from $D=260 \mathrm{~nm}(\sigma /\langle w\rangle=0.052)$ to $D=$ $3550 \mathrm{~nm}(\sigma /\langle w\rangle=0.027)$. This trend is attributed to less inter-column-competition with increased $D$ and therefore increased inter-column-spacing $d$. The growth of an adjacent nanocolumn will be more affected by irregularities in the growth of one nanocolumn if the seeds of
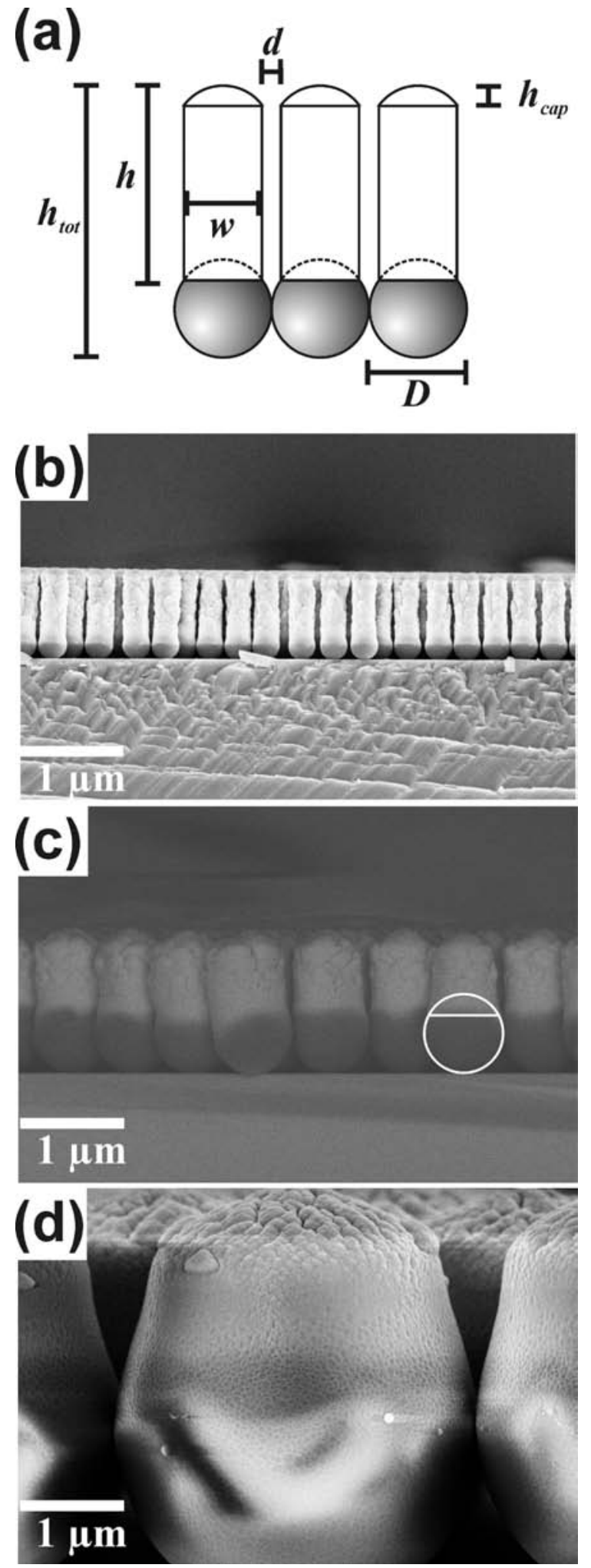

Fig. 2. (a) Illustration of the parameters used in the text. (b)-(d) Cross sectional SEM micrograph of $\mathrm{Si}$ nanocolumns grown on nanospheres with (b) $D=260 \mathrm{~nm}$, (c) $D=760 \mathrm{~nm}$ [back-scatter SEM micrograph to clearly show the fraction of the PS sphere (dark) that is covered by the Si column (bright)] and (d) $D=3550 \mathrm{~nm}$. The sketched sphere in (c) shows the nanosphere diameter and the fraction of the sphere actually covered with $\mathrm{Si}$. 
Table I. Relations between sphere diameter $D$, total structure height $h_{\text {tot }}$, column height $h$, fraction of the nanosphere diameter covered with Si. $f$, mean nanocolumn diameter $\langle w\rangle$, standard deviation of the column diameter distribution $\sigma$, normalized standard deviation $\sigma /\langle w\rangle$, inter-columnseparation $d$, height of hemispherical cap $h_{\text {cap }}$ and $h-h_{\text {cap }}$ for deposition at RT.

\begin{tabular}{|c|c|c|c|c|c|c|c|c|c|}
\hline$D[\mathrm{~nm}]$ & $h_{\mathrm{tot}}[\mathrm{nm}]$ & $h[\mathrm{~nm}]$ & $f[\%]$ & $\langle w\rangle[\mathrm{nm}]$ & $\sigma[\mathrm{nm}]$ & $\sigma /\langle w\rangle$ & $d[\mathrm{~nm}]$ & $h_{\text {cap }}[\mathrm{nm}]$ & $h-h_{\text {cap }}[\mathrm{nm}]$ \\
\hline 0 & 650 & 650 & & & & & & & \\
\hline 260 & 860 & 675 & 0.29 & 230 & 12 & 0.052 & 30 & 81 & 594 \\
\hline 500 & 1055 & 730 & 0.29 & 424 & 19 & 0.045 & 76 & 130 & 600 \\
\hline 760 & 1400 & 835 & 0.25 & 678 & 29 & 0.043 & 82 & 215 & 620 \\
\hline 2550 & 3080 & 1390 & 0.33 & 2360 & 70 & 0.029 & 190 & 600 & 790 \\
\hline 3550 & 4200 & 1840 & 0.33 & 3210 & 96 & 0.027 & 340 & 790 & 1050 \\
\hline
\end{tabular}

the columns (i.e., the tops of the PS spheres) are more close to each other. On substrates patterned with smaller nanospheres, the distance between growing columns is small. Column competition and flux capturing by one column on expense of a neighboured column will be more severe then. For large $D$ values, the distance between growing nanocolumns is large enough to suppress strong inter-column-competition, hence the narrow distribution of $\langle w\rangle$ in this case.

The mean column diameter $\langle w\rangle$ and the inter-columndistance $d$ as function of the PS sphere diameter $D$ are shown in Figures 3(b) and (c). Both $\langle w\rangle$ and $d$ scale linear with $D$, with ratios $\langle w\rangle / D=0.92$ and, respectively, $d / D=0.09$. Obviously, it is possible to grow hcp arranged $\mathrm{Si}$ nanocolums with predictable diameter and inter-column-distances on self-assembled monolayers of PS nanospheres.

Figures 2(b-d) show cross sectional micrographs of nanocolumns grown on PS spheres with different $D$ values. It can be seen that the nanostructures growing on each nanosphere resemble cylindrically shaped columns that are terminated with a hemispherical cap. Figure 2(a) shows a sketch of the columns to underline the parameters that will be used in the following: $h_{\text {tot }}$ is the total height of PS sphere plus Si column grown on it, $h$ is the height of a nanocolumn, measured with back-scatter SEM micrographs like in Figure 2(c), and $h_{\text {cap }}$ is the height of the hemispherical cap that terminates the column.

On bare substrates, the nominal height of the growing nanocolumns is $h_{0}=650 \mathrm{~nm}$ for the chosen deposition conditions. On substrates patterned with PS spheres, the total height of the structures increases with increasing sphere diameter. Figure 3(a) shows the relation between $D$ and $h_{\text {tot }}$. The relationship is linear, with a ratio of $h_{\text {tot }} / D=1$. From the linear function, it follows that a seed point (i.e., an island the column starts to evolve from) with a diameter of $32 \mathrm{~nm}$ would result in the growth of a nanocolumn with $h_{0}=650 \mathrm{~nm}$. This seed point diameter fits well with the experimental finding that on plain substrates, the growth of $\mathrm{Si}$ nanostructures starts with fibers having diameters in the range of (20-30) $\mathrm{nm}$ that merge together to form the actual nanostructures as growth continues. ${ }^{11}$

The $h$ values for different nanosphere diameters $D$ are listed in Table I as well. It can be seen that although on unpatterned substrates the nanocolumn height is $650 \mathrm{~nm}$, and the amount of deposited material is the same, $h$ increases with increasing $D$, from $h=675 \mathrm{~nm}(D=$ $260 \mathrm{~nm})$ to $h=1840 \mathrm{~nm}(D=3550 \mathrm{~nm})$. This increase
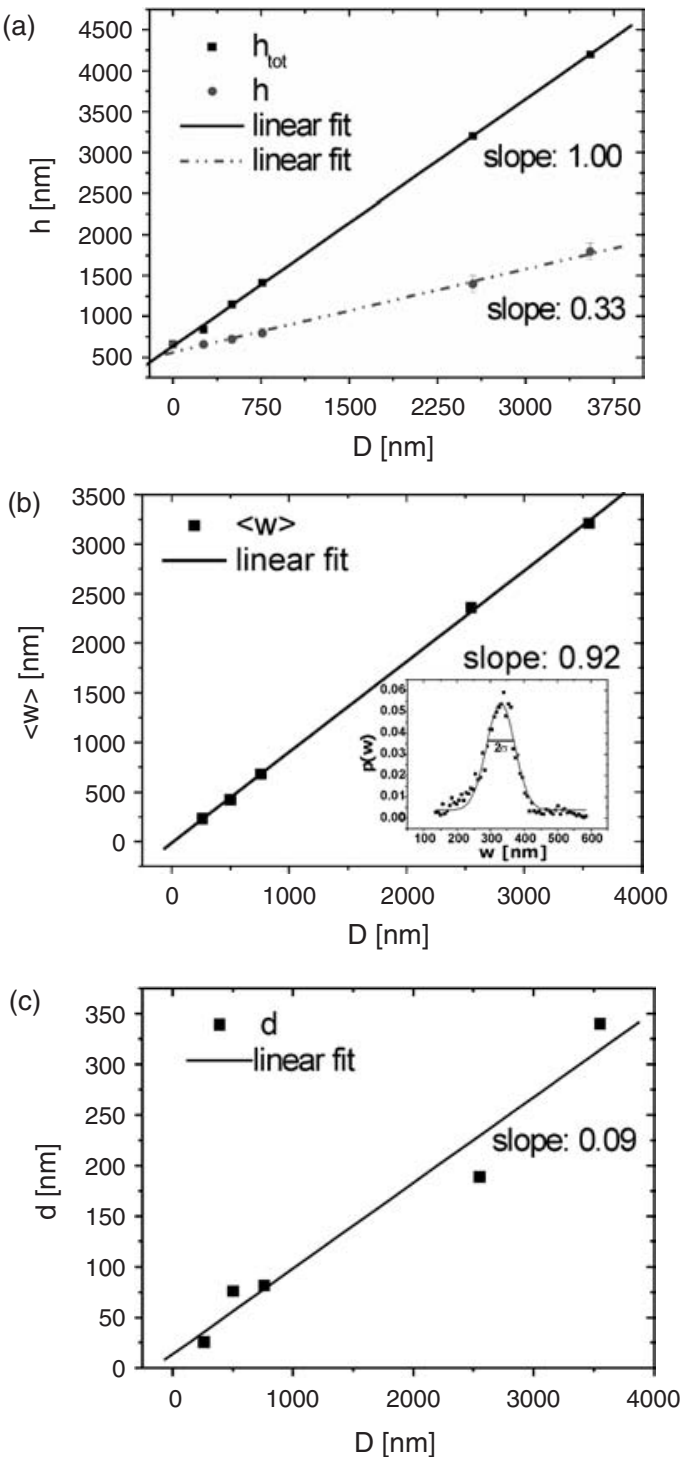

Fig. 3. Plots of (a) $h_{\text {tot }}$ and $h$, (b) the mean diameter of the nanocolumn $\langle w\rangle$, (c) the intercolumnar distance $d$, all as function of the nanosphere diameter $D$. Inset of (b): example of the Gaussian distribution of nanosphere diameters $\langle w\rangle$ for a set nanosphere diameter $D$ (here: $D=360 \mathrm{~nm})$. 
can be understood if the nanocolumn is seen as a cylinder, terminated with a hemispherical cap of height $h_{\text {cap }}$ on the side of the growth front and a hemispherical through on the other side, were the Si covers the top of the PS nanosphere. In this simple model, the volume of the column equals the volume of a cylinder with height $h-h_{\text {cap }}$, if one assumes the hemispherical cap to fit into the trough. As can be seen in Table I, up to $D=760 \mathrm{~nm}$, the values for $h-h_{\text {cap }}$ are approximately constant and with $h-h_{\text {cap }}=$ $(605 \pm 15) \mathrm{nm}$ close to the value $h_{0}=650 \mathrm{~nm}$ for unpatterned substrates. For $D=2550 \mathrm{~nm}$ and $D=3550 \mathrm{~nm}$, however, the values for $h-h_{\text {cap }}$ increase to $790 \mathrm{~nm}$ and $1050 \mathrm{~nm}$, respectively. This indicates that for larger sphere diameters, the assumption that the Si nanocolumn consists of a cap-terminated, dense cylinder with a hemispherical through on one side does not hold any more. As discussed above, the growth changes from single-column growth to multi-column growth on one sphere with increasing nanosphere diameter. Hence, for the multi-column case, the final $\mathrm{Si}$ column that consists of many, rod-like subcolumns that grow on one nanosphere is less dense than the single columns growing on nanospheres with smaller diameters, due to the intra-columnar voids between the rods. As the same amount of material is deposited, it follows that $h$ increases as well as $h-h_{\text {cap }}$.

The relationship between $D$ and $h$ is linear as well, as can be seen in Figure 3(a). The slope of the linear function is 0.33 in this case. This value is in good accordance with the fraction $f$ of the PS sphere that is actually covered with Si. In Table I, the $f$ values, ranging from $f=0.25$ to $f=0.33$ are listed.

\subsection{GLAD on $\mathrm{SiO}_{2}$ Nanospheres at Elevated $T_{\mathrm{S}}$}

To evaluate the temperature effect on the growth of $\mathrm{Si}$ nanocolumns on NSL patterned substrates, $\mathrm{SiO}_{2}$ nanospheres with $D=360 \mathrm{~nm}$ were used as seeds for the GLAD growth of Si nanocolumns at different $T_{\mathrm{S}}$. The results are shown in the micrographs of Figure 4 and in Table II.

At RT, the Si nanocolumns on unpatterned substrates have a mean diameter $\langle w\rangle_{0}=99 \mathrm{~nm}$ and a height of $h_{0}=700 \mathrm{~nm}$. With increasing substrate temperature, the nanocolumns on bare substrates increase their diameter, while their height decreases, until at $T_{\mathrm{S}}=350{ }^{\circ} \mathrm{C}$, some of the columns are broad enough to touch each other and eventually merge together, with $\langle w\rangle_{0}=167 \mathrm{~nm}$ and $h_{0}=570 \mathrm{~nm}$. This densification of the growing film with increasing $T_{\mathrm{S}}$ can be attributed to surface diffusion effects. ${ }^{16}$ At increased substrate temperatures, the seed number density in the beginning of the deposition is reduced, and therefore less nucleation sides for the GLAD growth are provided. Thus, fewer nanocolumns will start to grow and have more space to fill between them. Additionally, diffusion-driven mass transport at elevated temperatures attributes to column broadening as well as to an

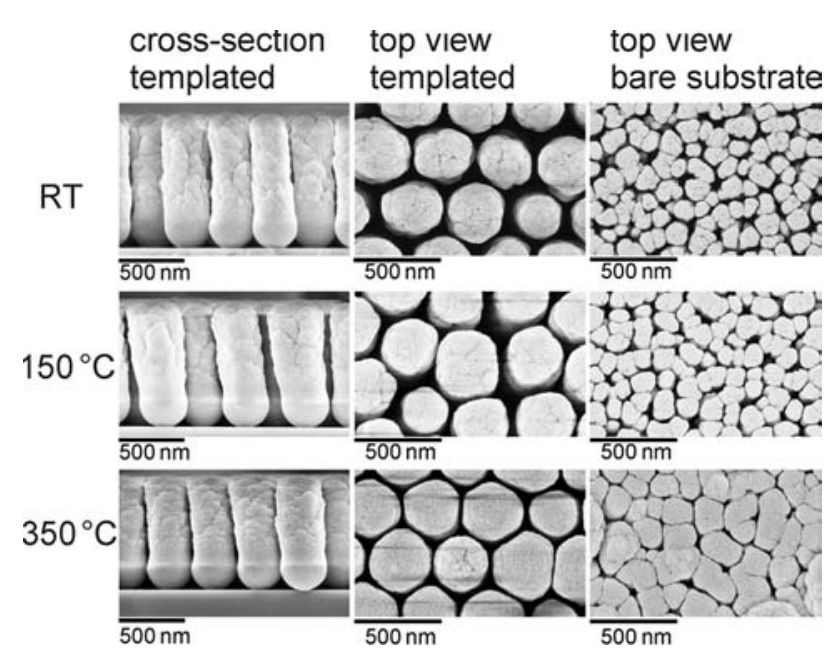

Fig. 4. Cross-sectional and top view micrographs of $\mathrm{Si}$ nanorods on $\mathrm{SiO}_{2}$ spheres with $D=360 \mathrm{~nm}$ and on unpatterned substrates at different substrate temperatures.

intra-columnar-densification, as voids in a single nanocolumn get filled more easily by $\mathrm{Si}$ particles when the surface diffusion is enhanced with increased $T_{\mathrm{S}}$. As a result, broad, densely packed nanocolumns that start touching each other will grow at elevated $T_{\mathrm{S}}$ on bare substrates, in contrast to the highly underdense films of well-separated nanocolumns that grow at RT.

In contrast to the growth on non-patterned substrates, the $\mathrm{Si}$ nanocolumns on $\mathrm{SiO}_{2}$ nanospheres do not show this extreme increase of $\langle w\rangle$ at elevated $T_{\mathrm{S}}$ found for the growth on unpatterned substrates. At RT, $\langle w\rangle=320 \mathrm{~nm}$. As $D=360 \mathrm{~nm},\langle w\rangle / D=0.89$. This is in good accordance with the constant $\langle w\rangle / D \approx 0.92$ which has been found for the growth of Si nanocolumns on PS spheres of different diameters. An increase of $T_{\mathrm{S}}$ induces an increase of $\langle w\rangle$ as in the case of the unpatterned substrate, see Table II. However, the increase of $\langle w\rangle$ is only slight when compared to the increase of $\langle w\rangle_{0}$ with increased temperatures. While with $\langle w\rangle\left(350{ }^{\circ} \mathrm{C}\right)=340 \mathrm{~nm}$ as compared to $\langle w\rangle(\mathrm{RT})=320 \mathrm{~nm}$, the ratio $\langle w\rangle\left(350^{\circ} \mathrm{C}\right) /\langle w\rangle(\mathrm{RT})=$ 1.06 , on unpatterned substrates this ratio amounts to $\langle w\rangle_{0}$ $\left(350{ }^{\circ} \mathrm{C}\right) /\langle w\rangle_{0}(\mathrm{RT})=1.69$.

As in the case of the unpatterned substrate, an increase of $T_{\mathrm{S}}$ results in a decrease of the nanocolumn height $h$. If we assume the column to have the volume $V$ of a cylinder with height $h-h_{\text {cap }}$ and diameter $\langle w\rangle$, a decrease of this height at higher $T_{\mathrm{S}}$ should be compensated with an increase of $\langle w\rangle$ in order to keep $V$ constant. Let the elevated temperature be $T$. Thus, if $V(\mathrm{RT})=V(T)$, it follows that $\left(h-h_{\text {cap }}\right)_{T}=\left(\langle w\rangle_{\mathrm{RT}} /\langle w\rangle_{T}\right)^{2} *\left(h-h_{\text {cap }}\right)_{\mathrm{RT}}$. Therefore, we should get $\left(h-h_{\text {cap }}\right)_{150}{ }^{\circ} \mathrm{C}=617 \mathrm{~nm}$ and $\left(h-h_{\text {cap }}\right)_{350{ }^{\circ} \mathrm{C}}=602 \mathrm{~nm}$. At $T_{\mathrm{S}}=150{ }^{\circ} \mathrm{C}$, the measured value $\left(h-h_{\text {cap }}\right)=620 \mathrm{~nm}$ is close to the value as predicted above. However, if $T_{\mathrm{S}}$ is increased to $350{ }^{\circ} \mathrm{C}$, the measured value $\left(h-h_{\text {cap }}\right)=560 \mathrm{~nm}$ is less than the predicted value of $602 \mathrm{~nm}$. 
Table II. Relations between substrate temperature $T_{\mathrm{S}}$, total structure height $h_{\text {tot }}$, column height $h$, mean nanocolumn diameter $\langle w\rangle$, standard deviation of the column diameter distribution $\sigma$, normalized standard deviation $\sigma /\langle w\rangle$, inter-column-separation $d$, height of hemispherical cap $h_{\text {cap }}$, $h-h_{\text {cap }}$, height of nanocolumn on unpatterned substrate $h_{0}$, mean nanocolumn diameter on unpatterned substrate $\langle w\rangle_{0}$, and normalized standard deviation on unpatterned substrate $\sigma /\langle w\rangle$.

\begin{tabular}{|c|c|c|c|c|c|c|c|c|c|c|c|}
\hline $\begin{array}{l}T_{\mathrm{S}} \\
{\left[{ }^{\circ} \mathrm{C}\right]}\end{array}$ & $\begin{array}{c}h_{\mathrm{tot}} \\
{[\mathrm{nm}]}\end{array}$ & $\begin{array}{c}h \\
{[\mathrm{~nm}]}\end{array}$ & $\begin{array}{c}\langle w\rangle \\
{[\mathrm{nm}]}\end{array}$ & $\begin{array}{c}\sigma \\
{[\mathrm{nm}]}\end{array}$ & $\sigma /\langle w\rangle$ & $\begin{array}{c}d \\
{[\mathrm{~nm}]}\end{array}$ & $\begin{array}{c}h_{\text {cap }} \\
{[\mathrm{nm}]}\end{array}$ & $\begin{array}{c}h-h_{\text {cap }} \\
{[\mathrm{nm}]}\end{array}$ & $\begin{array}{c}h_{0} \\
{[\mathrm{~nm}]}\end{array}$ & $\langle w\rangle_{0}$ & $(\sigma /\langle w\rangle)_{0}$ \\
\hline RT & 1060 & 760 & 320 & 23 & 0.072 & 40 & 80 & 680 & 700 & 99 & 0.25 \\
\hline 150 & 1000 & 715 & 336 & 22 & 0.065 & 24 & 95 & 620 & 610 & 117 & 0.23 \\
\hline 350 & 890 & 635 & 340 & 28 & 0.082 & 20 & 75 & 560 & 570 & 167 & 0.31 \\
\hline
\end{tabular}

This height decrease which is not accompanied by a strong column broadening as in the case of the unpatterned substrate can be attributed to an intra-columnar densification. At elevated substrate temperatures, the lengths scales for surface diffusion increase, and intra-columnar voids fill with diffusing adatoms more easily, which leads to a denser nanocolumn with decreased height as compared to columns grown at RT. On the other hand, as the seed points of the Si nanocolums (i.e., the top surfaces of the $\mathrm{SiO}_{2}$ spheres) are still well-separated, at $350{ }^{\circ} \mathrm{C}$ the adatom diffusion lengths are still too small for the incoming particles to overcome the distance between two $\mathrm{SiO}_{2}$ spheres. Therefore, no distinct column broadening or even merging of adjacent nanocolumns will appear between RT and $T_{\mathrm{S}}=350{ }^{\circ} \mathrm{C}$ on NSL patterned substrates due to a negligible inter-column-growth competition, in contrast to the case of an unpatterned substrate, where an increased surface diffusion length increases the average column width, which in turn leads to a strongly competitive growth mode that favors the survival of the broadest columns on expense of smaller ones.

\section{CONCLUSIONS}

Using NSL templated substrates with different nanosphere diameters, regular arrays of vertical $\mathrm{Si}$ nanocolumns with different diameters $w$ can be grown with GLAD. Those columns are cylindrically shaped, with a hemispherical cap on the growth front side. With increasing nanosphere diameter $D$, the growth changes from single-column growth to multiple-column growth on a single nanosphere. In the case of multiple-column growth (large $D$ ), the $\mathrm{Si}$ nanocolumn that consists of a number of sub-columns is less dense as in the case of single-column growth (small $D$ ). Consequently, since the amount of deposited material is constant, the nanocolumn height increases with increasing $D$. The mean diameter $\langle w\rangle$ of the Si nanocolumn scales linearly with the nanosphere diameter $D$, with $\langle w\rangle / D \approx 0.92$. Accordingly, the inter-column-separation $d$ scales linearly with $D$ as well, with $d / D \approx 0.09$, giving the ability to grow arrays of nanocolumns with defined inter-column-spacings.

Additionally, it was found that on unpatterned substrates, an increase of the substrate temperature $T_{\mathrm{S}}$ from
RT to $350{ }^{\circ} \mathrm{C}$ induces a morphology change from wellseparated nanocolumns with diameters in the range of $100 \mathrm{~nm}$ (for a column height of $700 \mathrm{~nm}$ ) to broad, merged columns with diameters in the range of $160 \mathrm{~nm}$. In contrast to that, increasing $T_{\mathrm{S}}$ yields only a moderate broadening of nanocolumns grown on $\mathrm{SiO}_{2}$ nanospheres, from $\langle w\rangle=320 \mathrm{~nm}(\mathrm{RT})$ to $\langle w\rangle=340 \mathrm{~nm}\left(350^{\circ} \mathrm{C}\right)$. This could be related with surface diffusion effects. This different, temperature-driven growth behaviour of $\mathrm{Si}$ nanocolumns on either templated or bare substrates could be used for size separation or microfluidic applications. ${ }^{17}$ If, for example, only a channel-like part of the substrate would be covered with nanospheres prior to the GLAD process, performing GLAD at $350{ }^{\circ} \mathrm{C}$ would result in a dense film, with only small pores intersecting broad nanocolumns on the unpatterned part of the substrate, whereas the patterned channel could be used as a sieving matrix, with pore sizes determined by the inter-column-separation and therefore by the nanosphere diameter.

Acknowledgments: This work is supported by the project P3 within the DFG research group FOR 522 "Architecture of nano- and microdimensional structure elements."

\section{References and Notes}

1. K. Robbie, L. J. Friedrich, S. K. Dew, T. Smy, and M. J. Brett, J. Vac. Sci. Technol. A 13, 1032 (1995).

2. C. M. Zhou and D. Gall, Thin Solid Films 515, 1223 (2006).

3. C. Patzig, B. Rauschenbach, W. Erfurth, and A. Milenin, J. Vac. Sci. Technol. B 25, 833 (2007).

4. J. J. Steele, A. C. van Popta, M. M. Hawkeye, J. C. Sit, and M. J. Brett, Sens. Actuators B 120, 213 (2006).

5. Q. H. Wu, L. de Silva, M. Arnold, I. J. Hodgkinson, and E. Takeuchi, J. Appl. Phys. 95, 402 (2004).

6. S. R. Kennedy, M. J. Brett, O. Toader, and S. John, Nano Lett. 2, 59 (2002).

7. B. Dick, M. J. Brett, T. J. Smy, M. R. Freeman, M. Malac, and R. F. Egerton, J. Vac. Sci. Technol. A 18, 1838 (2000).

8. S. V. Kesapragada, P. Victor, O. Nalamasu, and D. Gall, Nano Lett. 6, 854 (2006).

9. T. J. Yim, D. Y. Kim, S. S. Karajanagi, T. M. Lu, R. Kane, and J. S. Dordick, J. Nanosci. Nanotechnol. 3, 479 (2003).

10. T. Karabacek, J. P. Singh, Y. P. Zhao, G. C. Wang, and T. M. Lu, Phys. Rev. B 68, 125408 (2003).

11. E. Schubert, T. Höche, F. Frost, and B. Rauschenbach, Appl. Phys. A: Mater. Sci. Process. 81, 481 (2005). 
12. C. M. Zhou and D. Gall, Thin Solid Films 516, 433 (2007).

13. J. Rybczynski, U. Ebels, and M. Giersig, Colloids and Surfaces A: Physicochem. Eng. Aspects 219, 1 (2003).

14. E. Schubert, J. Fahlteich, T. Höche, G. Wagner, and B. Rauschenbach, Nucl. Instrum. Methods Phys. Res. B 244, 40 (2006).
15. Image Metrology, www.imagemet.com

16. C. Patzig and B. Rauschenbach, J. Vac. Sci. Technol. A 26, Accepted for publication.

17. G. K. Kiema, M. O. Jensen, and M. J. Brett, Chem. Mater. 17, 4046 (2005).

Received: 31 January 2008. Accepted: 11 March 2008. 